\title{
Apixaban bewährt sich im Versorgungsalltag
}

Zur Schlaganfallprophylaxe bei Pa-
tienten mit nicht-valvulärem Vorhofflim-
mern (VHF) werden im Praxisalltag ne-
ben Vitamin-K-Antagonisten (VKA) seit
einiger Zeit regelmäßig NOAK (nicht
Vitamin-K-abhängige orale Antikoagu-
lanzien) wie z. B. Apixaban (Eliquisø) ein-
gesetzt. Prof. Michael Näbauer, München,
zufolge wurde in den für die Zulassung
der NOAK in dieser Indikation relevan-
ten Studien als Vergleichssubstanz der in-
ternational am häufigsten verordnete
Wirkstoff Warfarin gewählt. In Deutsch-
land werde jedoch fast ausschließlich
Phenprocoumon verwendet.
„Angesichts dessen fordern Ärzte in
Deutschland seit Langem Untersuchun-
gen, in denen NOAK mit Phenprocou-
mon verglichen werden“, berichtete der
Kardiologe. Mit CARBOS, einer retro-
spektiven Datenbankanalyse, stehen
jetzt erstmals Daten zum Verträglich- keitsprofil von Apixaban zur Schlaganfallprophylaxe bei Patienten mit VHF aus dem deutschen Versorgungsalltag zur Verfügung [1]. Basis der Analyse bildeten anonymisierte Krankenversicherungsdaten von Patienten, die neu auf ein NOAK oder Phenprocoumon eingestellt worden waren.

\section{Weniger schwere Blutungsereignisse}

„Die Datenauswertung gibt Hinweise darauf, dass eine Therapie mit Apixaban im Vergleich zu einer Behandlung mit Phenprocoumon mit einem niedrigeren Risiko für schwere Blutungen (adjustierte Hazard Ratio [HR] 0,68; 95\%-Konfidenzintervall [KI] 0,51-0,91; $\mathrm{p}<0,008$ ), gastrointestinale Blutungen (adjustierte HR 0,53; 95\%-KI 0,39-0,72; p < 0,001) sowie Blutungen jeglicher Art (adjustiertes HR 0,80; 95\%-KI 0,70-0,92; $\mathrm{p}<$ $0,002)$ assoziiert ist", so Näbauer. Diese

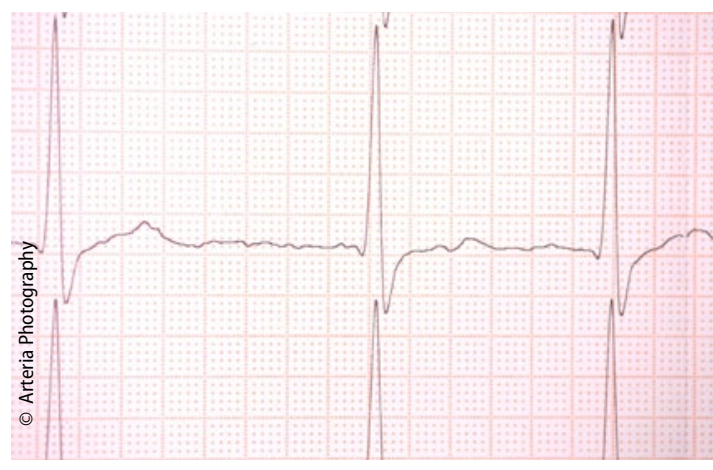

Vorhofflimmern - hier ist Gefahr in Verzug!

Daten aus dem Versorgungsalltag stützen damit wichtige Ergebnisse der klinischen Phase-III-Zulassungsstudie ARISTOTLE [Granger CB et al. N Engl J Med. 2011;365:981-92].

\section{Dr. Silke Wedekind}

- Pressegespräch „Aus der Klinik und aus dem deutschen Versorgungsalltag: Aktuelle Daten zu Apixaban in der Schlaganfallprophylaxe bei nicht-valvulärem Vorhofflimmern"; Rom, August 2016 (Veranstalter BMS, Pfizer)

\section{Hypertonie und Hyperlipidåmie}

\section{Dreifachkombination fördert Compliance}

— „In Deutschland weisen etwa zwölf Millionen Menschen sowohl eine Hyperlipidämie als auch eine Hypertonie auf“, betonte Prof. Ulrich Laufs, Homburg. Um das stark erhöhte kardiovaskuläre Risiko dieser Patienten zu senken, müssen beide Risikofaktoren konsequent angegangen werden.

Dies erfordert eine Mehrfachmedikation: Der Großteil aller Hypertoniker benötigt mindestens zwei Antihypertensiva für eine effektive Blutdruckkontrolle, und der Goldstandard zur Senkung erhöhter LDL-Cholesterin-Spiegel ist ein Statin. Eine hohe Tablettenlast wirkt sich jedoch negativ auf die Adhärenz aus: In einer Metaanalyse betrug die Adhärenz bei Einnahme einer Tablette pro Tag 79\%, bei Einnahme von vier Tabletten pro Tag nur noch 51\% [Clin Ther.
2001;23:1296-310]. Sechs Monate nach Beginn einer antihypertensiven und lipidsenkenden Therapie nimmt nur noch rund ein Drittel der Patienten die notwendigen Medikamente ein [Arch Intern Med. 2005;165:1147-52].

\section{Weniger Tabletten, mehr Kardioprotektion}

Eine compliancefördernde Therapie bietet die fixe Dreierkombination aus dem ACE-Hemmer Perindopril, dem Kalziumantagonisten Amlodipin und Atorvastatin $\left(\right.$ Triveram $\left.^{\circledast}\right)$. „Alle drei Wirkstoffe zeichnen sich durch eine 24-Stunden-Wirkung aus und haben in großen randomisierten kontrollierten Studien unter Beweis gestellt, dass sie die kardiovaskuläre Ereignisrate reduzieren“, erklärte Prof. Florian P. Limbourg, Han- nover. Dies bestätigte eine Subgruppenanalyse des Lipidarms der ASCOT-Studie [J Hypertens. 2014;32(e-suppl 1):e63. Abs 5B01]: Das Risiko für das Auftreten des kombinierten Endpunkts aus kardiovaskulärer Sterblichkeit, nicht-tödlichem Herzinfarkt und Schlaganfall war unter der Dreifachkombination um 42\% geringer als in der mit Atenolol/Thiazid/ Atorvastatin behandelten Vergleichsgruppe.

Vorteile könnte die neue Fixkombination vor allem für vorbehandelte Hypertoniker mit Hypercholesterinämie bieten, um die Compliance dieser Patienten zu verbessern.

Dr. Kirsten Westphal

- Pressegespräch "Triveram ${ }^{\varpi}-3$ Wirkstoffe, 2 Indikationen, 1 Tablette" am 16. September 2016 in München (Veranstalter: Servier) 